Communications in Physics, Vol. 24, No.3S2 (2014), pp. 63-70

DOI:10.15625/0868-3166/24/3S2/5061

\title{
THERANOSTIC GOLD NANOSHELLS: FROM SYNTHESIS TO IMAGING AND PHOTOTHERMAL THERAPY APPLICATIONS
}

\author{
NGHIEM THI HA LIEN, VU THI THUY DUONG, VU DUONG, \\ DO QUANG HOA, AND TRAN HONG NHUNG \\ Institute of Physics, Vietnam Academy of Science and Technology \\ E-mail: halien@iop.vast.ac.vn
}

Received 20 June 2014

Accepted for publication 20 August 2014

\begin{abstract}
Gold nanoshells (GNSS) were grown on monodispersed aminoprotpyltriethoxysilane (APTES) functionalized of silica nanoparticles (NPs) cores with varying sizes ranging from 40-180 nm synthesized by Stober route. Gold shells were deposited onto the surface of silica NPs by tetrakis(hydroxymethyl) phosphonium chloride (THPC) and electroless gold plating method. The coverage of the gold nanoshells on the surfaces of the silica NPs was evaluated using UV-VIS/NIR spectrospcopy and transmission electron microscopy (TEM). The plasmon resonance wavelengths of these gold nanoshells were tunable from visible to near infrared region. The GNSs were also bioconjugated with anti-HER2 monoclonal antibody for diagnostic breast cancer cells using dark field microscope technique. These GNS NPs play a role as nanoheaters transforming light to heat. With the present of these GNS NPs at volume density of $3.6 \times 10^{10} \mathrm{NPs.cm^{3 }}$ in chicken tissue samples, illuminated by $808 \mathrm{~nm}$ laser at the power density of $62 \mathrm{~W} . \mathrm{cm}^{2}$ the temperature of tissue sample reachs $110^{\circ} \mathrm{C}$ after 20 minutes illumination.
\end{abstract}

Keywords: gold nanoshells, silica NPs, SPR, dark field image, photothermal therapy.

\section{INTRODUCTION}

Gold nanoshells typically consist of a low dielectric spherical core nanoparticle, such as silica or polystyrene, coated with a thin layer of gold whose thickness ranges from a few nanometers to tens of nanometers. GNS NPs are plasmonic materials with intense absorbing and scattering properties. The surface plasmon resonance (SPR) of GNS NPs can be fine-tuned over the visible to near-infrared spectrum region by adjusting the relative core/shell ratio [1-3]. Moreover, the SPR effect of GNSs that operates within the spectral range of 700 to $900 \mathrm{~nm}$, matches with the optical requirements of tissue. Gold colloids or GNSs can also be conjugated with biological molecules. Therefore, the application of GNSs has been an increasing interest in the biomedical field, incorporating diagnostic and therapy research studies that include optical labeling for tumor cell imaging[3], controlled drug delivery, controlled gene delivery [4-6] and plasmonic photothermal therapy [7-9] .

(C)2014 Vietnam Academy of Science and Technology 
THERANOSTIC GOLD NANOSHELLS: FROM SYNTHESIS TO IMAGING AND PHOTOTHERMAL THERAPY ...

HER2 (human epidermal growth factor receptor 2) is a protein that is highly aggressive in breast cancers. In order to detect a tumor before it turns into late stage cancer, monoclonal antibody anti-HER 2 are used for diagnostic imaging.

Recent years, research relatting to physical property, heat generation by gold NPs under optical illumination (nanoheaters), has attracted much interest. The heat generation from these nanoheaters involves not only absorption of incident photons, but also the conversion of photon energy into heat energy as well as heat transfer from the NP to the surrounding matrix [10-13].

In this study, as an example of biospecific molecule binding, the GNS NPs were conjugated with the monoclonal antibody anti HER 2 using to identify the breast cancer cells BT747 by images of dark field microscopy. Especially, the temperature changes in chicken tissue containing the GNS NPs with size of $150 \mathrm{~nm}$ under CW diode laser illumination at $808 \mathrm{~nm}$ depending on GNS NPs concentration have been investigated. The results showed that the temperature of the chicken tissue samples can be reached to proximity $950 \mathrm{C}$ to $1100 \mathrm{C}$ at laser power of $62 \mathrm{~W} . \mathrm{cm}^{-2}$ after 20 min. illumination with average volume density of GNS NPs of $1.2 \times 10^{10}$ to $3.6 \times 10^{10} \mathrm{NP}^{-\mathrm{cm}^{-3}}$. These results demonstrated the role of GNS NPs in cancer theranostic.

\section{EXPERIMENTAL SECTION}

\section{II.1. Reagents}

Tetraethylorthosilicate (TEOS, 99\%), ammonium hydroxide (NH4OH, $29 \%$ in water), and glutathione (GSH) were purchased from Sigma-Aldrich. APTES (99\%); ethanol, tetrachloroauric acid trihydrate $\left(\mathrm{HAuCl}_{4} .3 \mathrm{H}_{2} \mathrm{O}, 99.9 \%\right)$, THPC $(80 \%$ solution in water), sodium hydroxide $(\mathrm{NaOH}, 99 \%)$, trisodium citrate dihydrate $\left(\mathrm{Na}_{3} \mathrm{C}_{6} \mathrm{H}_{5} \mathrm{O}_{7} \cdot 2 \mathrm{H}_{2} \mathrm{O}, 99 \%\right)$, potassium carbonate (99 $\%$ ), and formaldehyde (37\% solution in water) were purchased from Merck. Mouse anti-HER2 IgG monoclonal antibody (280003z) was $1 \mathrm{mg} / \mathrm{mL}$ in $1 \%$ BSA solution was purchased from Invitrogene. Deionized water was used in all experiments.

\section{II.2. Preparation of gold nanoshells}

GNSs were prepared by seeds and growth method and detailed in previous our work [14], using silica core with diameter of 40-180 nm synthesized by Stober route. The particle surface was then terminated with amine groups by reaction with APTES. Very small THPC gold NPs (1-2 nm) was prepared the method of Duff et al. The size and stability of THPC gold were clearly improved by using citrate buffer of $4.6 \mathrm{mM}$. $1 \mathrm{ml}$ of APTES functionalized of silica NPs were then added to $3 \mathrm{ml}$ of THPC gold NPs solution. THPC gold NPs absorb to the amine groups on the silica NPs surface resulting in a APTES functionalized silica NP decorated with discrete of THPC gold NPs, called the seeds NPs. The 10-20 nm gold shell layers were then grown on these seeds via the electroless gold plating process by using of gold plating solution in the presence of formaldehyde. The GNS surfaces were coated with GSH to avoid the GNS NPs aggregation. The GNS NPs were also bioconjugated with anti HER2 IgG monoclonal antibody which is specific molecules for diagnostic breast cancer cells. An amount of HER2/BSA antibody was added into the above GNS NPs solution to generate HER2/BSA antibody coated GNS NPs. The process was done by stirring the solution for 1 hour at room temperature till optically clear. To remove the excess free bio-molecules from anti HER2 IgG GNS NPs solution, anti HER2 IgG-GNS NPs complex was then washed thrice with Diwater via centrifugation of $2000 \mathrm{rpm}$ at ambient temperature for 15 minutes. The anti HER2 IgG-GNSs complex was redispersed in water before use. 


\section{II.3. Characterization of gold nanoshells}

The absorption spectra (300-1100 nm) were recorded by JASCO V-5700 spectrophotometer. Transmission electron microscopy (TEM) images of the silica NPs and GNSs were obtained from JEOL JEM 1011 microscope at the acceleration voltage of $80 \mathrm{kV}$. The samples were prepared by drop coating onto an amorphous carbon film supported by a 200-mesh copper grid. The light scattering images of GNS NPs were observed by Nikon Ti-E microscopy.

\section{II.4. Applications of Gold Nanoshells}

\section{Photothermal experimental}

The GNSs with core size of $140 \mathrm{~nm}$ and $15 \mathrm{~nm}$ gold shell were dispersed in water to the volume density of $7.7 \times 10^{10}$ particles. $\mathrm{mL}^{-1}$. Chicken tissues with the dimensions of $4 \times 4 \times$ $4 \mathrm{~mm}^{3}$ were prepared to study the photothermal effect. The solution of GNS NPs were slowly injected to the tissue sample at $2 \mathrm{~mm}$ depth under the surface of the samples. These tissue samples were illuminated by NIR laser $(808 \mathrm{~nm}$ ) for $20 \mathrm{~min}$. and on a excitation spot (1 $\mathrm{mm}$ in diameter). The power of excitation beam is $62 \mathrm{~W} . \mathrm{cm}^{-2}$. Temperature profiles were monitored by a sensitive temperature sensor PT 100. The experiments were performed at room temperature of $29^{\circ} \mathrm{C}$.

\section{Imaging experimental}

The anti HER2 IgG-GNS NPs complex was incubated with a cultural breast cancer BT747 for 2 hours. This complex strongly binds at high affinity to epidermal growth factor receptor 2 antigens on cell surfaces. The excess of anti HER2 IgG-GNS NPs complex was removed via centrifugation of $2000 \mathrm{rpm}$ at ambient temperature. The light scattering images of BT747 cell labeling with GNSs NPs were observed on a Nikon Ti-E via dark field mode.

\section{RESULTS AND DISCUSSION}

\section{III.1. Characterization of gold nanoshells}

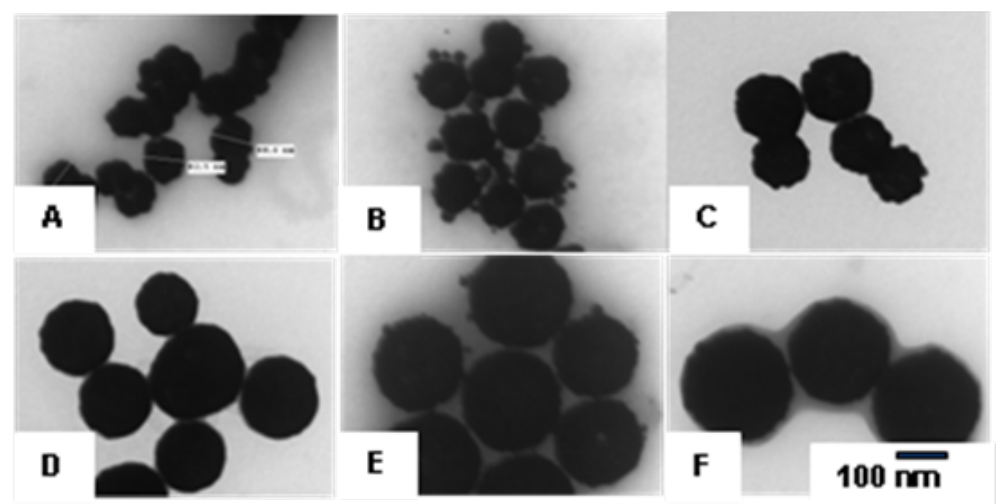

Fig. 1. TEM images of GNSs formatted from varying silica NPs core sizes (40-180nm from A-F) with the thickness of gold shells at about $15 \mathrm{~nm}$. The same scale bar (100nm) applies to all images.

The Fig. 1 shows the TEM images of the GNSs respective silica core NPs (Fig. 1 A-F) with the corresponding sizes of $40 \pm 3 \mathrm{~nm}, 65 \pm 5 \mathrm{~nm} ; 80 \pm 5 \mathrm{~nm} ; 110 \pm 5 \mathrm{~nm}, 140 \pm 5 \mathrm{~nm}$, and 
$180 \pm 5 \mathrm{~nm}$. The coverage was uniform regardless of silica NPs core sizes. The thickness of gold shell, following complete coverage, was determined about of $15 \mathrm{~nm}$.

\section{a) Optical characterization of gold nanoshells}

Fig. 2 shows the SPR absorption spectra of the silica-gold core-shell grown from the prepared THPC-gold-decorated APTES-functionalized silica NPs seed particles. Increasing the surface gold coverage on the silica NPs, with a specific core size, presents a red shift from 580 to $870 \mathrm{~nm}$. A secondary peak appears in the shorter wavelength region of a broad spectra (Fig 2a). The red shifts were no longer apparent when the surface coverage was complete. Further gold deposition, which results in the increase of the shell thickness, conversely causes a blue shift of the SPR absorption peak with more intense secondary peak at the shorter wavelengths. The use of silica NPs with variable core sizes - whereby surface gold coverage was completed at the thickness of $15 \mathrm{~nm}$ - also induced a shift in the SPR absorption spectra (Fig. 2b). These results show that the SPRs of the prepared silica-gold core-shell NPs are strongly dependent on both the extent of surface gold coverage on the silica core NPs and the size of the core silica NPs (at a given surface gold coverage). Hence, by accordingly adjusting these two properties, it is possible to tailor the SPR absorption to the $550-1000 \mathrm{~nm}$ spectral region, which is ideal for optical bio-imaging and plasmonic photothermal therapy applications, thereby making these nanoshells suitable for biomedical applications.
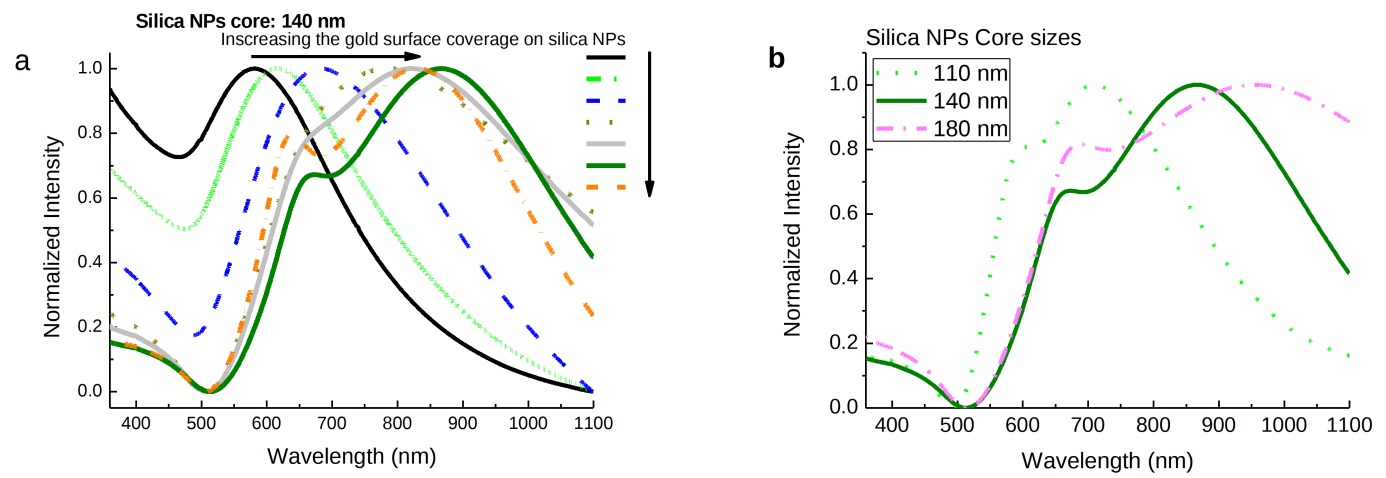

Fig. 2. Absorption spectra of silica-gold core-shell NPs with (a) varying surface gold coverage (diameter of silica core: $140 \mathrm{~nm}$ ) and (b) varying silica NPs core sizes (complete gold coverage; thickness of gold nanoshell: $15 \mathrm{~nm}$ ).

\section{b) Functionalization of gold nanoshells}

Fig. 3(a) shows the SPR absorption spectra of silica-gold core-shell NPs with sizes of 100 $\mathrm{nm}$ before (green line) and after (orange line) being coated with GSH. After being coated with GSH, the SPR absorption spectrum of GNS NPs solution is slightly red shift (2 nm), and the absorbance mostly has no change. As show in figure 3B, the red shift about $12 \mathrm{~nm}$ in SPR absorption spectra was observed with GNSs coated by monoclonal antibody anti HER2 compared to that of bare GNSs. These results correspond with our previous studies [15] which indicated that the protein monoclonal antibody IgG HER2 are well conjugated with GNS NPs. 

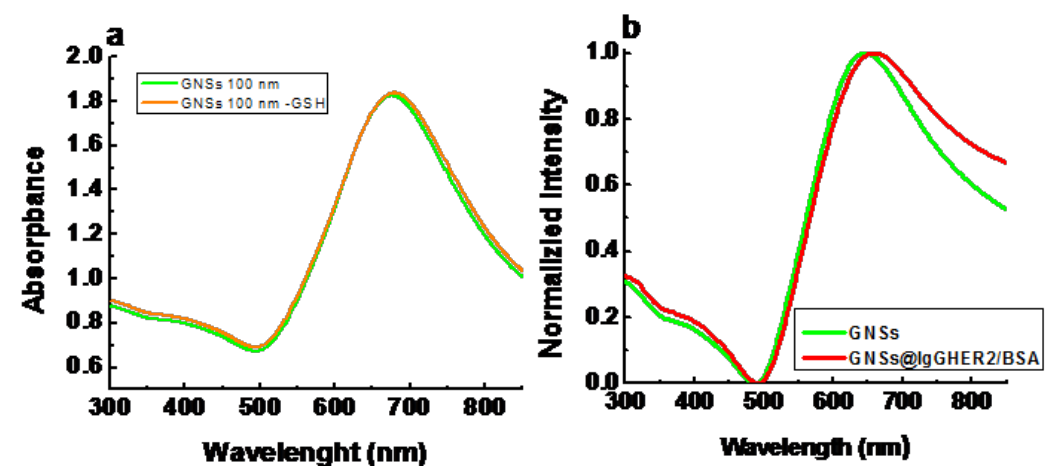

Fig. 3. a) SPR absorption spectra of GNSs NPs with sizes of 100nm before (green color) and after (orange color) coated GSH. b) SPR absorption spectra of GNSs NPs with sizes of $100 \mathrm{~nm}$ before (green color) and after (red color) coated with monoclonal antibody antiHER2.

Fig. 4 presents the characterizations of the sample as nanoheaters which were used for the investigation of light to heat conversion. Fig. 4(a) is the images dark field microscopy of the GNS NPs. The GNS NPs strongly scatter orange color associated by the surface plasmon oscillation of gold shells. This monocolor of dark field microscopy images indicated that the GNS NPs are very monodispersed. SPR absorption spectrum of these nanoheaters was showed in Fig. 4B, the peak position broad from 700 to $1100 \mathrm{~nm}$ where we can chose the wavelength of laser illumination.
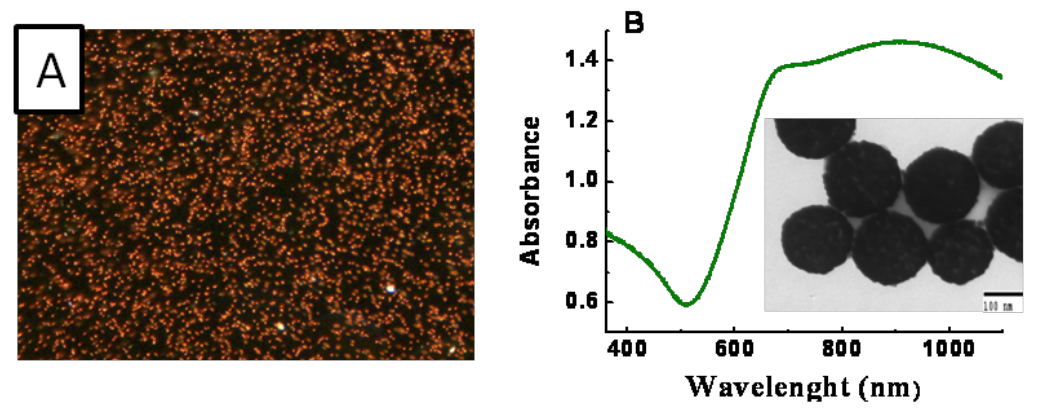

Fig. 4. Dark field microscope (A), and SPR spectrum (B) of GNS NPs

The GNS NPs with core sizes varying from 40-180 nm and gold shell thickness of 10-20 $\mathrm{nm}$ have been prepared, giving the peak position of the SPR ranging from 550 to $1000 \mathrm{~nm}$. The monoclonal antibody IgG HER2 functionalized GNSs NPs targeting to breast cancer cells was successfully prepared.

\section{III.2. Theragnostic of gold nanoshells}

\section{a) Photothermal therapy of GNSs}

Fig. 5(a) show us the results of the light to heat conversion of GNS NPs in chicken tissues samples were illuminated by a continuous diode laser at wavelength of $808 \mathrm{~nm}$ and at laser 
power of $62 \mathrm{~W} . \mathrm{cm}^{-2}$ after $20 \mathrm{~min}$. illumination. The green star points and red triangle points are the temperature profiles corresponding with the $1.2 \times 10^{10}$ to $3.6 \times 10^{10}$ particle $/ \mathrm{cm}^{3}$ in two the chicken tissue samples with dimensions of $4 \times 4 \times 4 \mathrm{~mm}^{3}$. These results presented the temperature of chicken tissue samples attain to $95^{\circ} \mathrm{C}-110^{\circ} \mathrm{C}$ corresponding with the present two above GNS NPs amounts and below 400C when absence of GNS NPs (data not shown). This light to heat conversion process can be explained following the energy balance equation model as detailed in ref of Richardson et al. [11], in which the temperature profile of the samples, after laser is turned on, is given by

$$
T(t)=T_{0}+\frac{A}{B}(1-\exp (-B t)) \text { when } A \neq 0
$$

where $A\left({ }^{\circ} \mathrm{C} / \mathrm{s}\right)$ is the rate of energy absorption, $B$ is the rate constant associated with heat loss $\left(\mathrm{s}^{-1}\right), T_{0}$ is ambient temperature and $T(t)$ is temperature at instant $t$.

The rate constant of heat loss $\mathrm{B}$ are determined from the obtained measurement results in Fig. 5A, by following the temperature decay back to the ambient temperature $\left(T_{0}\right)$ after laser excitation turned off (when $A=0$ ) by relation $\ln \left(T(t)-T_{0}\right) /\left(T_{\max -T_{0}}\right)$ versus time, as plots are showed in Fig. 5B. Here, $T_{\max }$ is used the temperature value at 20 minutes when laser was turned off. The rate constant $B$ for heat loss to be $0.01 \mathrm{~s}^{-1}$, which was determined from the slops of these linear fits, as showed in solid lines on Fig. 5B.
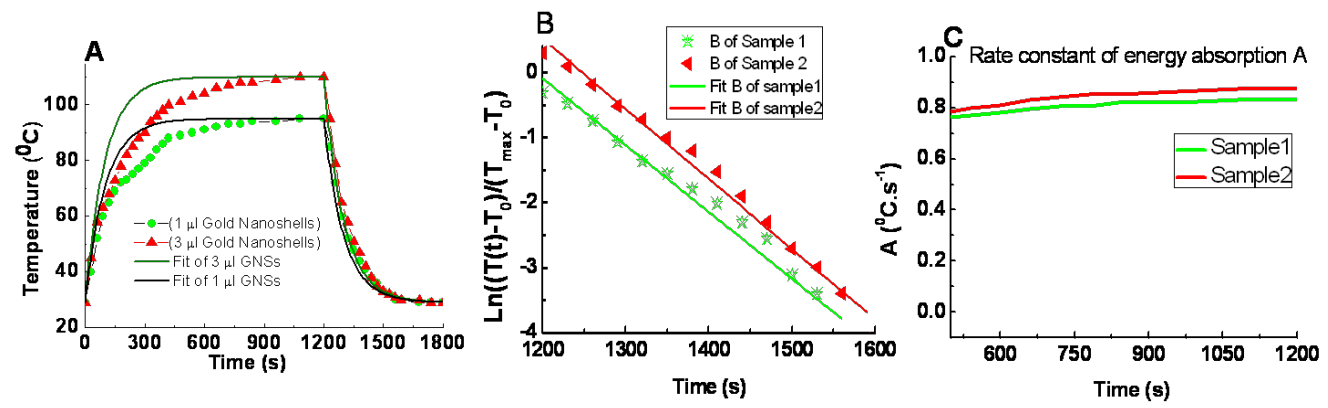

Fig. 5. The light to heat conversion of GNSs with illumination power laser of $62 \mathrm{~W} . \mathrm{cm}^{-2}$ at wavelength of $808 \mathrm{~nm}$ vs. time. The solid lines correspond to energy balance model of Richardson et al. [11] (A). Plotting of the $\ln \left(T(t)-T_{0}\right) /\left(T_{\max }-T_{0}\right)$ as function of time after the laser is turned off (B) illumination with two different GNSs amounts in chicken tissue samples. The rate constants of energy absorption A corresponding with two above samples (C).

Using obtained values of the rate constant $\mathrm{B}$, we can deduced the values of the rate of energy absorption $\mathrm{A}$ at steady-state (showed in figure 5C) from our experimental measurements, which is respectively varied from 0.669 to $0.809\left({ }^{\circ} \mathrm{C} / \mathrm{s}\right)$ chicken tissue samples with $1 \mu \mathrm{L}$ and $3 \mu \mathrm{L}$ GNSs, in this case, with relation $A=B *\left(T_{S S}-T_{0}\right) /(1-\exp (-B . t))$. Using both above values of rate of energy absorption $A$ and heat loss $\mathrm{B}$, we can recast the temperature profiles using the energy balance model in Eq. 1, which are plotted in the solid lines as showed in Fig. 5A. As seen in the Fig. 5A, when laser cut-off $(A=0)$, the observed data match well with the model fit lines. The characteristic time to establish thermal equilibrium in tissue samples headed nonuniformly by a laser beam is $\Delta(t)_{d i f f}=L^{2} / D_{w}[10]$ where $L$ and $D_{w}$ are a dimension of sample and thermal 
diffusivity of water (considering the water dominated in chicken tissue). With $L=0.4 \mathrm{~cm}$ and $D_{w}=1.4 \times 10^{-3} \mathrm{~cm}^{-2} / \mathrm{s}$, we obtain $\Delta t_{\text {diff }}=114 \mathrm{~s}$, which is higher than $B^{-1}=100 \mathrm{~s}$. Therefore, this explains why at the first times the temperature of tissue samples is slowly attained thermal equilibrium. These also may be due to the GNS NPs are nonuniformly in the tissue samples and the cross section of samples is larger than cross sectional area of thermo couple wires. These results from the energy balance equation model fit matches well with our nanoheaters-GNSs. Therefore, we can use it to design the light to heat conversion process for our GNSs for any amounts or any density power of laser.

\section{b) Imaging dark field microscopy of breast cancer cells labeling by GNSs}

The Fig. 6 shows the dark field images of the breast cancer cells BT-747 labeling with the complex GNS-anti HER2. On this picture, we can see the GNS NPs attached well on the BT 747 cell surfaces. Thank to the light scattering of GNS NPs we can clearly observed BT 747 cells with microscopy by dark field as show in Fig. 6. These results show that monoclonal antibody IgG anti HER2 was well conjugated with GNS NPs, and the ability of the GNS@IgG-HER2 antibody complex as cancer labeling. The results show that this complex is able to detect the target cells.

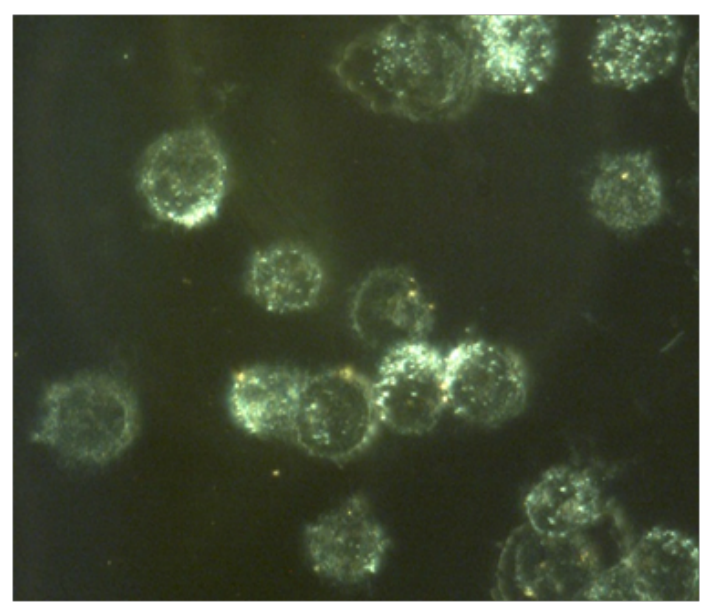

Fig. 6. Images dark field microscopy of breast cancer cells BT-747 recognized by the complex GNS-anti HER2

The investigation of the photothermal effect of GNS NPs in chicken tissue under nearinfrared illumination demonstrates that GNSs has a high light to heat conversion efficiency. The temperature of chicken tissue samples reach $110 \mathrm{oC}$ in the present of GNS NPs and bellow $40 \mathrm{oC}$ when absence of the GNS NPs, illuminated by $808 \mathrm{~nm}$ laser at power density $I=62 \mathrm{~W} / \mathrm{cm}^{2}$ after 20 min illumination. The complex GNS@HER2 was applied for imaging the BT474 cells. These results confirmed that the GNS NPs are able to use for optical bio-imaging and plasmonic photothermal therapy applications, thereby making these GNS NPs suitable for biomedical applications. Clinical trials for photothermal tumor ablation using laser-excited tunable plasmonic nanoparticles already underway, increasing understanding of the efficacy of plasmonic nanoparticle-based photothermal heating takes on increased urgency. 


\section{CONCLUSIONS}

Gold shells with tunable thickness were growth on silica with varying average diameter ranging from 40-180 nm, using a low temperature mediated route. These results show that the SPRs of the prepared silicagold coreshell NPs are strongly dependent on both the extent of surface gold coverage on the silica core NPs and the size of the core silica NPs (at a given surface gold coverage). Hence, by accordingly adjusting these two properties, it is possible to tailor the SPR absorption to the $550-1000 \mathrm{~nm}$ spectral region, which is ideal for optical bio-imaging and plasmonic photothermal therapy applications, thereby making these nanoshells suitable for biomedical applications. The GNS NPs were coated by targeted bio-molecules such as monoclonal antibody IgG HER2. The light scattering of GNS NPs labeling breast cancer cells BT 747 obtained by dark field microscopy that proved their ability in recognize the breast cancer cells. The investigation of the photothermal effect of GNS NPs in chicken tissue under near-infrared illumination demonstrates that GNS NPs has a high light to heat conversion efficiency. The temperature of chicken tissue samples reach $110^{\circ} \mathrm{C}$ in the present of GNS NPs with volume density of $3.6 \times 10^{10} \mathrm{NPs}_{\mathrm{cm}} \mathrm{cm}^{-3}$ illuminated by $808 \mathrm{~nm}$ laser at power density $I=62 \mathrm{~W} / \mathrm{cm}^{2}$ after 20 min illumination.

\section{ACKNOWLEDGEMENT}

This study was supported by Nafosted Grant No. 103.06-2010.10. Authors would like to thank the Important Laboratory on Photonics (VAST), which provided the facilities for this study.

\section{REFERENCES}

[1] S. J. Oldenburg, R. D. Averitt, S. L. Westcott, and N. J. Halas, Chem. Phys. Lett. 288(2) (1998) 243-247.

[2] T. Pham, J. B. Jackson, N. J. Halas, and T. R. Lee, Langmuir, 18 (12) (2002) 4915-4920.

[3] C. Loo, A. Lowery, N. Halas, J. West, and R. Drezek, Nano Lett. 5(4) (2005) 709-711.

[4] G. B. Braun, A. Pallaoro, G. Wu, D. Missirlis, J. A. Zasadzinski, M. Tirrell, and N. O. Reich, ACS Nano, 3(7)(2009) 2007-2015.

[5] D. Pissuwan, T. Niidome, and M. B. Cortie, J. Controlled Release 149(1) (2011) 65-71.

[6] E.-K. Lim, E. Jang, K. Lee, S. Haam, and Y.-M. Huh, Pharmaceutics 5(2) (2013) 294-317.

[7] H. Liu, D. Chen, F. Tang, G. Du, L. Li, X. Meng, W. Liang, Y. Zhang, X. Teng, and Y. Li, Nanotechnology 19(45) (2008) 455101.

[8] S. Lal, S. E. Clare, and N. J. Halas, Accounts Chem. Res. 41(12) (2008) 1842-1851.

[9] L. Hirsch, R. J. Stafford, J. A. Bankson, S. R. Sershen, B. Rivera, R. E. Price, J. D. Hazle, N. J. Halas, and J. L. West, Proc. Natl. Acad. Sci. 100(23) (2003) 13549-13554.

[10] D. K. Roper, W. Ahn, and M. Hoepfner, J. Phys. Chem. C 111(9) (2007) 3636-3641.

[11] H. H. Richardson, M. T. Carlson, P. J. Tandler, P. Hernandez, and A. O. Govorov, Nano Lett.9 (3) (2009) 11391146.

[12] J. R. Cole, N. A. Mirin, M. W. Knight, G. P. Goodrich, and N. J. Halas, J. Phys. Chem. C 113(28) (2009) 12090-12094.

[13] B. Khlebtsov, V. Zharov, A. Melnikov, V. Tuchin, and N. Khlebtsov, Nanotechnology 17(20) (2006) 5167-5179.

[14] T. H. L. Nghiem, T. N. Le, T. H. Do, T. T. D. Vu, Q. H. Do, and H. N. Tran, J. Nanoparticle Res. 15(11) (2013) 1-9.

[15] T. H. L. Nghiem, T. H. La, X. H. Vu, V. H. Chu, T. H. Nguyen, Q. H. Le, E. Fort, Q. H. Do, and H. N. Tran, $A d v$. Nat. Sci. Nanosci. Nanotechnol. 1(2) (2010) 025009 